\title{
Decreased ability in the segregation of dynamically changing vowel-analog streams: a factor in the age-related cocktail-party deficit?
}

\author{
Pierre Divenyi ${ }^{1,2 *}$ \\ 1 Department of Music, Center for Computer Research in Music and Acoustics, Stanford University, Stanford, CA, USA \\ ${ }^{2}$ Speech and Hearing Research, Veterans Affairs Northern California Health Care System, Martinez, CA, USA
}

Edited by:

Elyse S. Sussman, Albert Einstein

College of Medicine, USA

\section{Reviewed by:}

Mounya Elhilali, Johns Hopkins

University, USA

Torsten Rahne, Universitätsklinikum

Halle (Saale), Germany

Jennifer Lentz,

Indiana University, USA

\section{*Correspondence:}

Pierre Divenyi, Center for Computer Research in Music and Acoustics, Stanford University, 360 Lomita

Court, Stanford, CA 94305, USA

e-mail:pdivenyi@

ccrma.stanford.edu
Pairs of harmonic complexes with different fundamental frequencies f0 (105 and $189 \mathrm{~Hz}$ or 105 and $136 \mathrm{~Hz}$ ) but identical bandwidth $(0.25-3 \mathrm{kHz})$ were band-pass filtered using a filter having an identical center frequency of $1 \mathrm{kHz}$. The filter's center frequency was modulated using a triangular wave having a $5-\mathrm{Hz}$ modulation frequency $\mathrm{f}_{\text {mod }}$ to obtain a pair of vowel-analog waveforms with dynamically varying single-formant transitions. The target signal $S$ contained a single modulation cycle starting either at a phase of $-\pi / 2$ (up-down) or $\pi / 2$ (down-up), whereas the longer distracter $N$ contained several cycles of the modulating triangular wave starting at a random phase. The level at which the target formant's modulating phase could be correctly identified was adaptively determined for several distracter levels and several extents of frequency swing $(10-55 \%)$ in a group of experienced normal-hearing young and a group of experienced elderly individuals with hearing loss not exceeding one considered moderate. The most important result was that, for the two f0 differences, all distracter levels, and all frequency swing extents tested, elderly listeners needed about $20 \mathrm{~dB}$ larger $\mathrm{S} / \mathrm{N}$ ratios than the young. Results also indicate that identification thresholds of both the elderly and the young listeners are between 4 and $12 \mathrm{~dB}$ higher than similarly determined detection thresholds and that, contrary to detection, identification is not a linear function of distracter level. Since formant transitions represent potent cues for speech intelligibility, the large $\mathrm{S} / \mathrm{N}$ ratios required by the elderly for correct discrimination of single-formant transition dynamics may at least partially explain the well-documented intelligibility loss of speech in babble noise by the elderly.

Keywords: speech perception, aging, auditory scene analysis, formants, frequency modulation

\section{INTRODUCTION}

In aging various auditory functions of the individual are often impaired. Perhaps the most disturbing aspect of this impairment is the significantly reduced ability to understand speech in social noise or a reverberant environment, commonly referred to as the loss of the "cocktail-party effect" (CPE). Although, expectedly, this deficit is exacerbated by presbycusic - the typical age-related sensorineural high-frequency elevation of auditory thresholds (Carhart and Tillman, 1970) - it is often also experienced by elderly individuals with normal audiograms or having, at worst, a mild-to-moderate hearing loss (Dubno et al., 1984; Divenyi and Haupt, 1997; Snell et al., 2002). Causes of the CPE deficit in the elderly are complex. On the peripheral end, hearing loss has been for long known to affect speech understanding in babble noise, regardless of age (Humes et al., 1994). On the other end of the spectrum, age-related cognitive decline has also been implicated, be it decreased selective attention to concurrent speech (Sommers, 1997), impaired short-term recall of words (Murphy et al., 2000), or reduced working memory capacity (Ng et al., 2013). These factors are among those recognized to increase in the mental effort required when the elderly listens to speech in a CPE setting (Zekveld et al., 2011). But, between peripheral and cognitive extremes there is a host of sensory/nervous system processes indispensable for understanding speech in interference that are also deficient. One group of these are deficits of temporal processing in diverse time ranges, such as gap detection and discrimination necessary for the perception of stop consonants and affricates (Snell and Frisina, 2000), duration discrimination (Fitzgibbons and Gordon-Salant, 1994) affecting accurate perception of subsyllabic and syllabic segments, temporal modulation transfer functions ( $\mathrm{He}$ et al., 2008) and resistance to modulation interference (Bacon and Takahashi, 1992; Humes et al., 2013), formant transition discrimination (Elliott et al., 1989), and temporal-order discrimination (Fitzgibbons and Gordon-Salant, 1998). A second group is related to localization, which is also known to be impaired in aging (Herman et al., 1977; Abel et al., 2000). This impairment makes CPE performance poorer by reducing or altogether canceling the 2.5 -to- $4 \mathrm{~dB}$ release from masking provided by spatial separation of the target and the interference (Ihlefeld and Shinn-Cunningham, 2008). 
From a strictly auditory standpoint, CPE can be regarded as an instance of masking with target speech as the signal and interference as the noise. However, since in speech the respective frequency ranges of the target and the interference seldom perfectly overlap, the rules derived from decades' worth of tonein-noise energetic masking research will be applicable only to specific speech segments. As proposed by authors investigating CPE in laboratories across different continents (Brungart et al., 2001; Cooke et al., 2008) the overwhelming portion of speech in babble noise the masking is informational, due to the similarity of the target and the interference (Lee and Richards, 2011). Research over the last few decades uncovered many aspects of informational masking (Watson, 1987; Lutfi, 1990; Kidd et al., 1994; Oh and Lutfi, 1998; Freyman et al., 2004) and was able to quantitatively specify the differences between the two modes of masking (Arbogast et al., 2002; Brungart and Simpson, 2002; Durlach et al., 2003b). As to informational masking in aging, some studies have shown elderly listeners to be more affected than the young (Freyman et al., 2008; Rajan and Cainer, 2008), while others found no age differences (Agus et al., 2009; Ezzatian et al., 2011). The disagreement between these results may stem from the lack of uniformly accepted definition of informational masking other than being different from energetic masking-a tautology pointed out by Durlach (Durlach et al., 2003a) — but also from the inherent difficulty of controlling for the ensemble of physical parameters of speech. However, the lack of age effect could also be due to the elderly listener using his/her experience to compensate for a low speech-to-noise ratio by relying on predictions derived from overlearned patterns (Divenyi, 2005).

CPE can be also viewed as the instance of auditory scene analysis (ASA, Bregman, 1990) most important for verbal communication. In fact, in a CPE situation the listener must continuously segregate a speech target stream from the babble stream or streams. While young normal-hearing individuals are able to understand speech in CPE settings even under quite unfavorable signal-to-noise ratios (SNR's), the SNR elderly individuals require is significantly higher (Gelfand et al., 1988; Snell et al., 2002), even when these individuals suffer from no or only mild presbycusic hearing loss (Divenyi and Haupt, 1997). The way ASA understands speech segregation of target from non-target speech is that harmonics of the fundamental frequency (f0) each of the simultaneous voices are grouped, thereby allowing the listener to focus on the harmonics of the target voice alone-as demonstrated by experiments on the segregation of non-speech harmonic complexes (Micheyl and Oxenham, 2010) and synthesized as well as natural speech sounds (Darwin et al., 2003; Roman and Wang, 2006; Lavandier and Culling, 2008). Segregation of concurrent vowels (Assmann and Summerfield, 1990), or speech of concurrent talkers (Darwin et al., 2003), is easier when their f0's are widely separated (e.g., as in the voices of different gender talkers) and becomes increasingly difficult as the difference between f0's decreases. Temporal asynchrony of vowels (Darwin and Hukin, 1998) or words (Lee and Humes, 2012) also facilitates their segregation. The ability to segregate one vowel in an ensemble of concurrent vowels increases when the f0 of one or several in the ensemble is modulated by a low-frequency sinusoid (i.e., when it undergoes a vibrato) (McAdams, 1990). In rooms, the target and non-target talkers are in spatially separated locations allowing the auditory system to segregate them, as shown in binaural experiments (Brungart and Simpson, 2002, 2007; Hawley et al., 2004).

But, looking from a broad perspective, speech is a dynamic signal characterized by constant changes. The changes can be defined in various ways, such as on the level of acoustics (fluctuating envelope, fundamental frequency variations, formant transitions, etc.), articulatory phonetics (gestural movements), descriptive phonetics and phonology (sequences and clusters of phonemic and sub-phonemic units), or higher-order linguistics (sequences of morphemes, words, word strings, sentences, sentence strings). Although computational characterization, and modeling, of these changes is nearly impossible at the higher levels of analysis, a mathematical formulation of the transform of acoustic signals to activity patterns observed at the cortical level, the complex modulation spectrum based on Gabor's wavelet transform (Gabor, 1946) has been gaining acceptance. Although Gabor conceived it for the reduction of information "atoms" in audio (i.e., telephone) communication, the transform and its inverse have been widely used for the analysis and synthesis of images (Levi and Stark, 1983) before being adopted for the analysis of audio signals (Pitton et al., 1996) and to models of the auditory system beyond peripheral analysis (Kowalski et al., 1996). Recognizing that both the temporal and spectral envelopes of natural (i.e., complex) sounds contain peaks that change over time, the transform represents the spectrum of these peaks as modulations in the temporal (rate, in $\mathrm{Hz}$ ) and spectral (scale in cycles per octave) domains. By choosing appropriate parameters for this model, called the "spectro-temporal receptive field" (STRF) model, it has been demonstrated that auditory cortex activity in the ferret (Chi et al., 2005) or in the song bird (Singh and Theunissen, 2003), as well as temporal-parietal cortical responses recorded with an electrode grid placed on the surface of patients awaiting epilepsy surgery (Mesgarani et al., 2008), can be fairly accurately modeled using this transform. In agreement with Plomp (1983)_ “... speech is a signal slowly varying in amplitude and frequency" - and with the $4-\mathrm{Hz}$ major mode of the temporal modulation spectrum of speech (Greenberg et al., 2003), shown to be language-independent (see e.g., Arai and Greenberg, 1997) speech input to this model shows that the predominant temporal modulation rate is slow and so is the scale of frequency peak shifts during relatively stable segments (e.g., vowels, fricatives, nasals, Elliott and Theunissen, 2009). Because the transform effectively uncovers patterns and features of complex signalsspeech, music, animal sounds, and environmental sounds - it has been used as a tool for the separation of concurrent auditory streams in a cocktail-party situation (Elhilali and Shamma, 2008; Mesgarani et al., 2011). Continuing this line of thought, if the auditory system uses temporal and spectral modulations to picture our acoustic world and to separate auditory objects, then studying the perception of signals modulated in amplitude and/or frequency, as well as its impairments, should bring us closer to the understanding of the success and failures of listening in a CPE setting. Thus, data on modulation detection/discrimination interference (MDI) in the amplitude (Moore et al., 1995; Moore and Sek, 1996), or frequency (Lyzenga and Carlyon, 1999) domains 
not only reveal parametric limitations of the Gabor transform applied to audition but also quantitatively describe the dynamic temporal and spectral map inside the existence limit of the CPE.

The present study continues the above line of reasoning in a set of experiments aimed at better understanding components of the deficiency elderly individuals display when listening to speech in the presence of speech interference. Since an earlier study showed the effect of duration and velocity on the perception of vowel transitions (Divenyi, 2009), and since the perception of frequency transitions in aging has been shown to correlate with intelligibility (Gordon-Salant et al., 2007), the experiments were focused on the way, and the extent to which, identification of a transition of interest is affected by the presence of an similar transition. The experiments used a single-formant simplified analogs of a target vowel and of an interfering vowel, each having a fixed fo and a formant peak modulated in frequency. A similar stimulus configuration was used in studies by Lyzenga and Carlyon (1999, 2005) focused on the effects of the difference between either modulating or fundamental frequency, and of spectral content. In contrast, the question the present experiments addressed was the target-todistracter ratio (TDR) necessary for a formant peak modulation pattern to be identified by normal-hearing young, and by elderly listeners without appreciable hearing loss.

\section{MATERIALS AND METHODS}

Stimuli in the experiments consisted of pairs of harmonic complexes: a target stream presented simultaneously with an interfering distracter stream. The 800 -ms distracter stream started $200 \mathrm{~ms}$ before the 400-ms target stream. The two streams had different fundamental frequencies (f0), one always $107 \mathrm{~Hz}$ for one of the streams and either 136 or $189 \mathrm{~Hz}$ for the other, thereby producing two different fundamental frequency separations $(\Delta \mathrm{f} 0)$, one wide $(\Delta \mathrm{f} 0 / \mathrm{f} 0=0.77$, approximately corresponding to the minor seventh musical interval) and one narrow $(\Delta \mathrm{f} 0 / \mathrm{f} 0=0.27$, approximately corresponding to the major third). At each $\Delta \mathrm{f} 0$ separation, the higher fo was assigned to the target in half of the conditions while it was assigned to the distracter in the other half. The spectrum of both streams contained only harmonics inside the 250- to $3000-\mathrm{Hz}$ band. Because this constraint resulted in a certain degree of difference between the perceived salience of the two streams, Terhardt's algorithm (Terhardt et al., 1982) was used to generate streams the salience of the dominant temporal ("virtual") pitch of which was comparable. Both streams were spectrally shaped to produce single-formant pseudo-vowels by passing them through second-order band-pass filters with a $6 \mathrm{~dB} /$ octave falloff, i.e., filters with formant characteristics not unlike that of natural vowels. The target stream's formant frequency $\mathrm{F}_{\mathrm{T}}$ was held constant at $1 \mathrm{kHz}$ during the first and last $100 \mathrm{~ms}$ of its duration, while during the central $200 \mathrm{~ms}$ the $\mathrm{F}_{\mathrm{T}}$ was modulated with a $5-\mathrm{Hz}$ triangular wave that went for $100 \mathrm{~ms}$ in one direction and for $100 \mathrm{~ms}$ in the other, thereby creating formant trajectory patterns in which $\mathrm{F}_{\mathrm{T}}$ was changing either updown or down-up starting from, and returning to, an $\mathrm{F}_{\mathrm{T}}$ of $1 \mathrm{kHz}$, with a maximum formant swing of $\Delta \mathrm{F}_{\mathrm{T}} \mathrm{Hz}$. In the distracter stream, the formant frequency $\mathrm{F}_{\mathrm{D}}$ was also modulated with a $5-\mathrm{Hz}$ triangular wave, except that the modulator waveform was during the whole duration of the distracter, creating a continuous

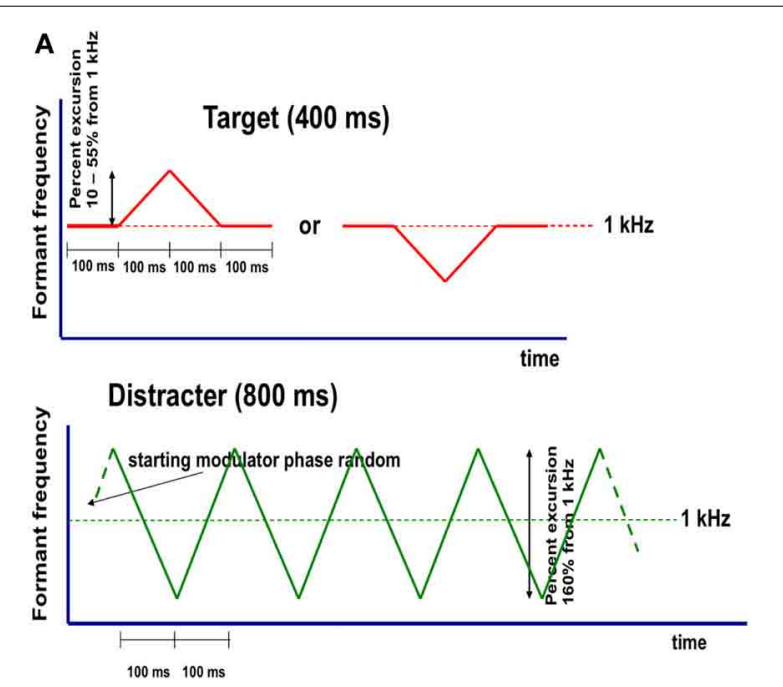

B Target with 55\% FM Swing plus Distracter, SNR=10 dB

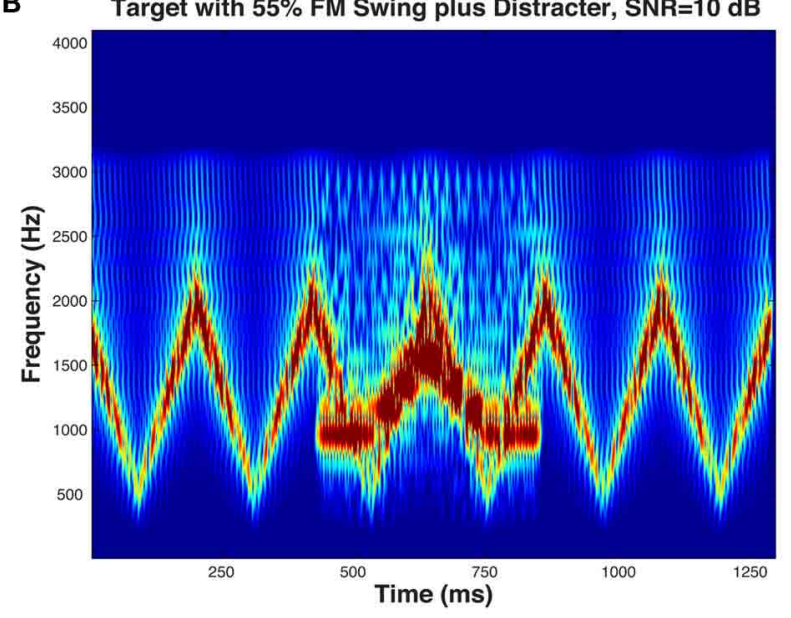

FIGURE 1 | (A) Schematic stimulus diagram, illustrating trajectory of the single formant's peak in the target and in the distracter,

frequency-modulated using a $5-\mathrm{Hz}$ triangular wave. The upper traces illustrate the two target formant peak trajectory patterns that the listeners had to identify: a 100-ms ascending followed by a 100-ms descending trajectory or a 100-ms descending followed by a 100-ms ascending trajectory, both trajectories flanked by two 100-ms steady-state portions with a constant formant peak at $1 \mathrm{kHz}$. The lower trace illustrates the trajectory of the distracter that consisted of an 800-ms pattern of repeating $100-\mathrm{ms}$ ascending and descending trajectories between the 525 and $1800-\mathrm{Hz}$ formant peak minimum and maximum that are equidistant from the $1-\mathrm{kHz}$ center on a basilar membrane distance scale. The starting phase of the distracter's modulating waveform varied randomly from trial to trial. All formant trajectories were linear on this distance scale. Both the target and the distracter were gated using a 25-ms cosinusoidal window to ensure that the onset and the offset of both were smooth. (B) Spectrogram of a trial. The target is an up-down transition with an FM excursion reaching $1550 \mathrm{~Hz}$, i.e., $55 \%$ higher than the resting $1-\mathrm{kHz}$ formant frequency. The SNR of the example shown, $10 \mathrm{~dB}$, is larger than most conditions used in the experiments.

up-down-up-down pattern. The extent of the distracter's formant trajectory was also larger than that of the target: the top and the bottom formant frequency extremes were 1800 and $525 \mathrm{~Hz}$, i.e. two frequencies equally distant from $1-\mathrm{kHz}$, a frequency at the 

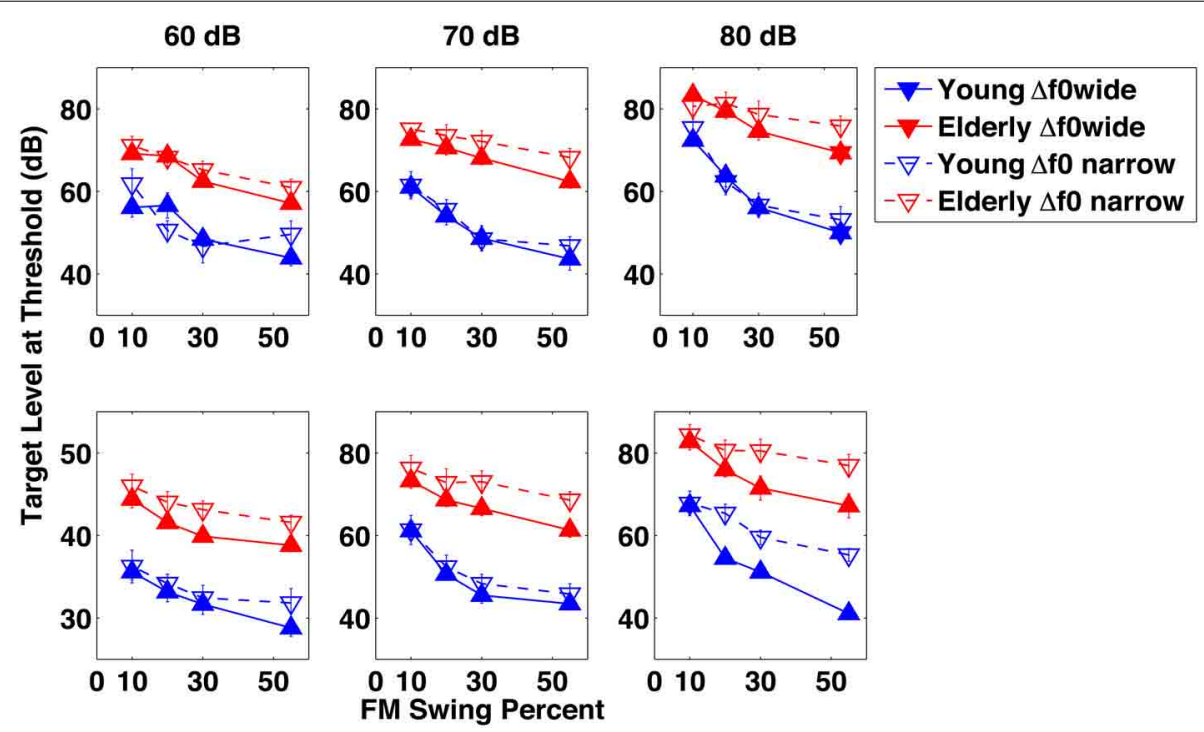

FIGURE 2 | Results of Experiment 1. Target level at identification threshold in $\mathrm{dB}$ as a function of the target formant's frequency modulation swing expressed as percent maximum displacement from $1 \mathrm{kHz}$, across three distracter levels in $\mathrm{dB}$, in separate columns of graphs. The elderly group's data are in red and those of the young group in blue. Filled symbols represent results for conditions in which the fundamental frequency separation $\Delta \mathrm{fO} / \mathrm{fO}$ between the target and the distracter was wide $(0.77)$, whereas data marked by the empty symbols are for conditions with a narrow (0.27) $\Delta \mathrm{fO} / \mathrm{fO}$. In the top row of graphs the data shown represent for conditions in which the fundamental frequency fo of the target was always lower than that of the distracter, whereas in the bottom row of graphs they represent conditions in which the fundamental frequency fo of the target was always higher than that of the distracter. center of the low and high extremes on Greenwood's (1962) scale of basilar membrane distances. Schematic formant frequencyvs.-time diagrams of the target and the distracter are shown in Figure 1A, with an audio example presenting the target, the distracter, and their combination. Throughout the experiments, the starting modulation phase of the distracter randomly varied from trial to trial. Figure 1B displays a spectrogram representing a trial having an up-down target a large, 55\%, formant excursion embedded in the distracter; the TDR is $+10 \mathrm{~dB}$ - a ratio larger than most used in the experiments proper and is shown here mainly for illustrative purposes.

The study included two experiments. The objective of the first was to examine how the ensemble of stimulus parameters affected the threshold of discriminability of formant transition patterns. The objective of the second experiment was to examine the effect of the stimulus parameters on the threshold of detectability (i.e., audibility) of the target. In Experiment 1, the subject performed a single-interval two-alternative forced choice task that consisted of identifying whether the formant trajectory pattern in the target stream was up-down or down-up, while ignoring the distracter stream. In each block of trials $\Delta \mathrm{F}_{\mathrm{T}} / \mathrm{F}_{\mathrm{T}}$, the frequency excursion (i.e., the swing) of the target, remained fixed at 10, 20, 30, or 55 percent and the overall level of the distracter was held constant at 60,70 , or $80 \mathrm{~dB}$ SPL. The difference between the fundamental frequencies of the target and the distracter, $\Delta \mathrm{f} 0 / \mathrm{f} 0$, was narrow or wide and varied from condition to condition, and so did the assignment of the higher or the lower of the fundamental frequencies to the target stream (and the other fundamental frequency to the distracter). In Experiment 2 the subject performed a two-interval two-alternative task in which he/she had to detect whether the target formant pattern was present in the first or the second interval, with the distracter being presented in both intervals. Two formant swing extents, 10 and 55 percent, were investigated with the distracter level constant at 60,70 , or $80 \mathrm{~dB}$ SPL. The higher fundamental frequency was always assigned to the target and the fundamental frequency separation was always the wide one $(\Delta \mathrm{f} 0 / \mathrm{f} 0=0.77)$.

Stimuli were digitally stored and delivered by a PC computer using an Echo Gina analog converter system connected to Tucker and Davis Technology filters and digital attenuators, and delivered diotically to headphones (Sennheiser SH 250). In each run of trials in both experiments the level of the target stream was varied adaptively from a starting point of $90 \mathrm{~dB}$ SPL to track the $79.4 \%$ correct performance threshold. The initial step size was $5 \mathrm{~dB}$ and was reduced with whenever the subject gave three consecutive correct responses first to 2 , and then to $1 \mathrm{~dB}$. The run was terminated at the tenth reversal and threshold in each run was calculated as the average of the target's $\mathrm{dB}$ level at the last eight reversals. The threshold estimate for each subject and each condition was the arithmetic mean of thresholds obtained in six to eight runs.

Listener performance was assessed for subjects in two groups. The young group included 17 normal-hearing individuals between 19 and 29 years of age (average $22.0 \pm 3.4$ years). The elderly group included 12 elderly individuals between 61 and 82 years of age (average $69.0 \pm 6.7$ years) in Experiment 1, 10 of whom also participated in Experiment 2. Their hearing impairment, when present, was a mild-to-moderate presbycusic sensorineural loss; the mean of the group's pure-tone average thresholds between 0.5 and $4 \mathrm{kHz}$ was $19.3 \pm 14.2 \mathrm{~dB}$ SPL. Several 
Table 1 | ANOVA of target identification data.

\begin{tabular}{|c|c|c|c|c|c|}
\hline \multicolumn{6}{|c|}{ Analysis of variance } \\
\hline Source & Sum sq. & $d f$ & Mean sq. & $\boldsymbol{F}$ & Prob $>F$ \\
\hline SUB(Sgroup) & 58931.9 & 27 & 2182.7 & 77.92 & 0 \\
\hline Dlevel & 28,678 & 2 & 14,339 & 511.91 & 0 \\
\hline Trghi/Lo & 1941.7 & 1 & 1941.7 & 69.32 & 0 \\
\hline DfO & 622.8 & 1 & 622.8 & 22.23 & 0 \\
\hline Swing & 33,475 & 3 & 11158.3 & 398.36 & 0 \\
\hline Sgroup & 48032.7 & 1 & 48032.7 & 1714.79 & 0 \\
\hline SUB(Sgroup) $*$ Dlevel & 6475.1 & 54 & 119.9 & 4.28 & 0 \\
\hline SUB(Sgroup) $*$ Trghi/Lo & 2826.3 & 27 & 104.7 & 3.74 & 0 \\
\hline SUB(Sgroup) $*$ DfO & 737.6 & 27 & 27.3 & 0.98 & 0.5011 \\
\hline SUB(Sgroup) $*$ Swing & 6641.1 & 81 & 82 & 2.93 & 0 \\
\hline Dlevel $*$ Trghi/Lo & 485.4 & 2 & 242.7 & 8.66 & 0.0002 \\
\hline Dlevel $*$ DfO & 13.4 & 2 & 6.7 & 0.24 & 0.7869 \\
\hline Dlevel $*$ Swing & 685.6 & 6 & 114.3 & 4.08 & 0.0005 \\
\hline Dlevel $*$ Sgroup & 1161.3 & 2 & 580.7 & 20.73 & 0 \\
\hline Trghi/Lo $*$ DfO & 194.4 & 1 & 194.4 & 6.94 & 0.0085 \\
\hline Trghi/Lo $*$ Swing & 1982.1 & 3 & 660.7 & 23.59 & 0 \\
\hline Trghi/Lo $*$ Sgroup & 1292.2 & 1 & 1292.2 & 46.13 & 0 \\
\hline Df0 $*$ Swing & 386.9 & 3 & 129 & 4.6 & 0.0033 \\
\hline DfO $*$ Sgroup & 89.6 & 1 & 89.6 & 3.2 & 0.0739 \\
\hline Swing $*$ Sgroup & 956.2 & 3 & 318.7 & 11.38 & 0 \\
\hline Error & 32016.3 & 1143 & 28 & & \\
\hline & & 139 & & & \\
\hline
\end{tabular}

Factors: Sgroup—elderly/young groups; Dlevel—Distracter level; Targhi/Lo—fo of target higher or lower than fO of distracter; DfO-fO separation wide/narrow; Swing-Maximum of target formant's excursion.

elderly subjects had normal hearing and none had hearing loss exceeding $25 \mathrm{~dB}$ under $3 \mathrm{kHz}$, that is, in the frequency region of the stimuli. Subjects were tested individually in sessions that lasted $1 \mathrm{~h}$ a day. All subjects received training with stimuli used in both experiments. Data collection for each subject was started after he/she obtained a score of at least 95 percent correct in two contiguous 60 -trial runs using the largest formant excursion (55\%) without the distracter present, and a score of at least 80 percent correct in two 60-trial runs with the distracter present at $60 \mathrm{~dB}$ SPL, using the $55 \%$ formant excursion, and a constant target level of $80 \mathrm{~dB}$ SPL. Typically, young subjects needed oneand-half session to reach these criteria, whereas elderly subjects needed, on the average, two-and-half sessions. Subject testing procedures were fully consistent with experimental protocols approved by the V.A. Northern California Health Care System's Institutional Review Board.

\section{RESULTS}

\section{EXPERIMENT 1-IDENTIFICATION OF FORMANT TRAJECTORY PATTERNS}

Figure 2 illustrates the results of the experiments on the identification of a dynamically changing single- and formant target pattern in the background of a dynamically changing single-formant distracter. The figure represents threshold level of the target pattern for the average of the elderly (red lines and symbols) and the young (blue lines and symbols) subject groups, as a function of the extent of the target formant's excursion across the three distracter levels. All panels compare results of the wide $(\Delta \mathrm{f} 0 / \mathrm{f} 0=0.77$, approximately minor seventh, solid lines) and narrow ( $\Delta \mathrm{f} 0 / \mathrm{f} 0=0.27$, approximately major third, dashed lines) f0 separations. In the top panels the target's f0 is lower and in the bottom panels it is higher than that of the distracter.

Looking at the young group's data within each and across all of the four figure panels, several general observations can be made (lower target thresholds indicating better performance):

(1) Increasing distracter level from 60 to 70 and $80 \mathrm{~dB}$ resulted in increased target levels. For a $20 \mathrm{~dB}$ increase in the distracter level a $10.6 \mathrm{~dB}$ target level increase was required, suggesting that the target level was a compressed nonlinear function of the distracter level.

(2) Decreasing the excursion resulted in an increase of the target threshold, although substantial increase was seen mainly at the smallest (10\%) excursion extent. The difference between target levels at the easiest (55\%) and hardest (10\%) swing extents averaged across all conditions was large (16.2 dB). Because most of the target level increase occurred between the two smallest swings (10\% and $20 \%)$, the target level was an expansive nonlinear function of the formant excursion.

(3) At both f0 separations, when the target's f0 was lower than distracter's the task was easier, resulting in target thresholds $2.75 \mathrm{~dB}$ lower on the average across all conditions.

(4) The large f0 separation was easier than the narrow one, resulting in target thresholds $4.6 \mathrm{~dB}$ lower on the average across all conditions.

(5) At the threshold of identifiability, the TDR was $-22.40 \mathrm{~dB}$ at the easiest and $-4.61 \mathrm{~dB}$ at the hardest condition, that is, the level of the target was below that of the distracter even when identifiability of the target was most difficult.

The trend of the elderly subjects' data mirrors that of the young subjects. In general, the differences within the elderly group's data with regard to swing, f0 separation, and target f0 are comparable to, or somewhat smaller than, those exhibited by the young subject group. The target level-distracter level nonlinearity for the elderly is a little smaller than for the young: a $12.6 \mathrm{~dB}$ target level increase for a $20 \mathrm{~dB}$ distracter level increase. Averaged across conditions, the target level difference between the easiest and hardest swing conditions was $9.6 \mathrm{~dB}$, between the assignments of the higher fo to the target or to the distracter was $4.02 \mathrm{~dB}$, and between the two f0 separation was a mere $0.2 \mathrm{~dB}$. However, when comparing the elderly and the young groups' results, one striking feature appears: the elderly subjects' target levels are about $20 \mathrm{~dB}$ higher than those of the young subjects, in all experimental conditions. In other words, in order to identify the target formant pattern as up-down or down-up, elderly listeners needed a target intensity about $20 \mathrm{~dB}$ higher than the young, regardless of the condition. Expressing the results as TDR, average TDR for the young was $-22.4 \mathrm{~dB}$ in the easiest and -4.60 in the most difficult condition, whereas TDR for the elderly was -2.85 and $4.34 \mathrm{~dB}$, respectively, for the two difficulty degrees. [The easiest 

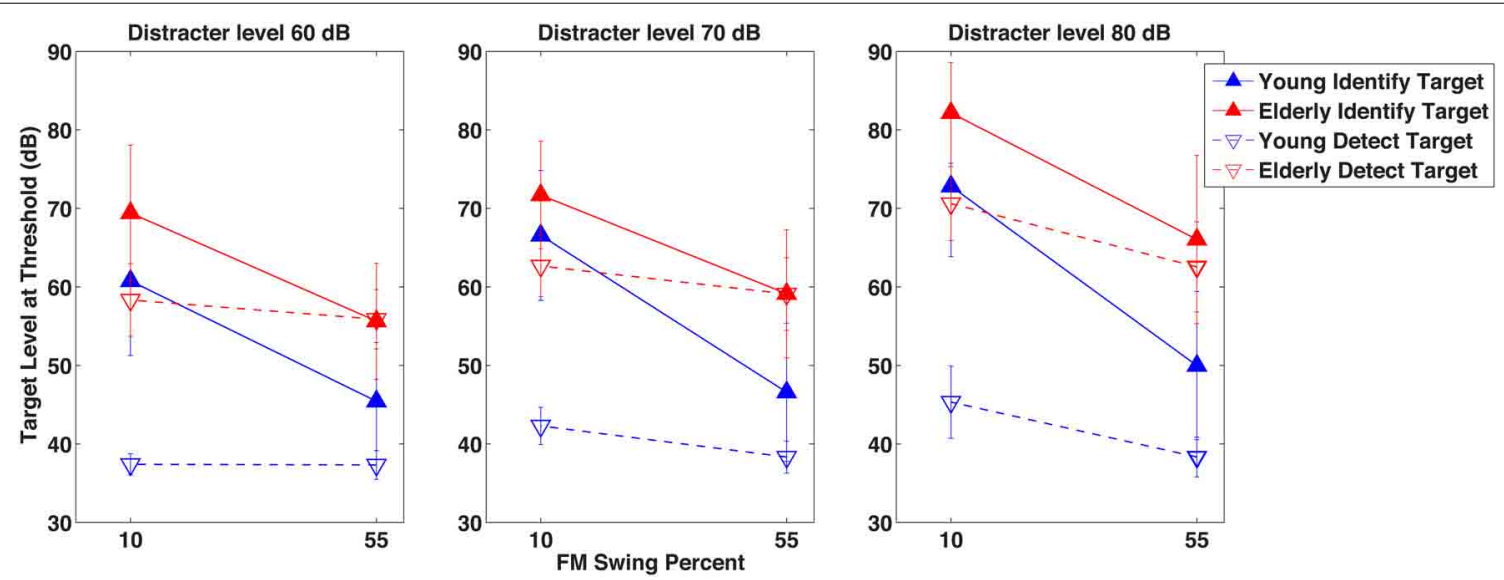

FIGURE 3 | Results of Experiment 2. A comparison of detection and identification of the target in the presence of the distracter. As in Figure 2, target level at threshold is shown in $\mathrm{dB}$ as a function of the target formant's FM excursion expressed as percent maximum displacement from $1 \mathrm{kHz}$. The three separate graphs indicate data for three distracter levels in $\mathrm{dB}$. Unfilled symbols represent threshold levels for the detection of the target, whereas filled symbols represent thresholds for the identification of the target. The fundamental frequency separation $\Delta \mathrm{fO} / \mathrm{fO}$ of the target and the distracter was wide $(0.77)$ and the fundamental frequency fo of the target was always higher than that of the distracter.
Table 2 | ANOVA of data from detection vs. identification tasks.

\begin{tabular}{|c|c|c|c|c|c|}
\hline \multicolumn{6}{|c|}{ Analysis of variance } \\
\hline Source & Sum sq. & $d f$ & Mean sq. & $\boldsymbol{F}$ & Prob $>F$ \\
\hline SUB(Sgroup) & 8922.3 & 25 & 356.9 & 11.86 & 0 \\
\hline Sgroup & 8352.9 & 1 & 8352.9 & 277.67 & 0 \\
\hline Dlevel & 285.8 & 2 & 142.9 & 4.75 & 0.0097 \\
\hline Det/ldent & 14427.4 & 1 & 14427.4 & 479.61 & 0 \\
\hline Swing & 2724.9 & 1 & 2724.9 & 90.58 & 0 \\
\hline SUB(Sgroup) $*$ Dlevel & 1855.4 & 50 & 37.1 & 1.23 & 0.1612 \\
\hline SUB(Sgroup) $*$ Det/ldent & 3781.2 & 25 & 151.2 & 5.03 & 0 \\
\hline SUB(Sgroup) $*$ Swing & 605.9 & 25 & 24.2 & 0.81 & 0.7318 \\
\hline Sgroup $*$ Dlevel & 223.2 & 2 & 111.6 & 3.71 & 0.0263 \\
\hline Sgroup $*$ Det/Ident & 19.3 & 1 & 19.3 & 0.64 & 0.4242 \\
\hline Sgroup $*$ Swing & 303 & 1 & 303 & 10.07 & 0.0018 \\
\hline Dlevel $*$ Det/ldent & 3686.5 & 2 & 1843.3 & 61.28 & 0 \\
\hline Dlevel $*$ Swing & 247.3 & 2 & 123.7 & 4.11 & 0.0179 \\
\hline Det/ldent $*$ Swing & 9287.4 & 1 & 9287.4 & 308.74 & 0 \\
\hline Error & 5535.1 & 184 & 30.1 & & \\
\hline Total & 60526.2 & 323 & & & \\
\hline
\end{tabular}

Factors: Sgroup—elderly/young groups; Dlevel_Distracter level; Det/ldentTask (detection vs. identification); Swing-Maximum of target formant's excursion.

condition was that of the $60 \mathrm{~dB}$ SPL distracter, the widest (55\%) swing, the wide $\mathrm{f} 0$ separation, and for the target having the higher f0 than the distracter. In the same vein, the hardest condition was that of the $80 \mathrm{~dB}$ SPL distracter, the narrowest (10\%) swing, the narrow f0 separation, and for the target having the lower f0 than the distracter]. Thus, the elderly-young discrepancy when the task is easy is the same $20 \mathrm{~dB}$ as for the overall data shown in the figures but it diminishes to only $8.3 \mathrm{~dB}$ when the tasks are difficult.
To uncover details and to analyze the statistics of the observations, an analysis of variance was conducted. Results of the ANOVA are shown in Table 1 . As the probability (p-) column indicates, all main effects—distracter level, formant swing extent, the size of f0 separation, and assignment of the higher f0 to target or distracter-were highly significant with $p=0.0001$, both within and across subjects. The subject group effect was also highly significant and so were the within subject and main effect interactions, except the within subject-f0 separation effect, indicating that some subjects found the task for both $\Delta \mathrm{fo} / \mathrm{f0}$ 's equally difficult or easy. The significant interaction between both individual subjects and subject group vs. the assignment of the higher fo to target or distracter indicates, as suggested by Figure 2, that the elderly, as well as some individual subjects, found it more consistently easier to identify the target pattern when the target f0 was the higher one. The significant interaction between formant swing extent and f0 assignment indicates, as both rows of graphs in Figure 2 illustrate, that the target's f0 assignment to the higher f0 made the task easier only when the swing was relatively large, i.e., when the task itself was less difficult. The lack of significance of the $\Delta \mathrm{f} 0 / \mathrm{f} 0$-distracter level and the $\Delta \mathrm{f} 0 / \mathrm{f0}$-subject group interactions indicate that frequency separation was a stable effect unaffected by the loudness of the distracter or the age of the listener.

\section{EXPERIMENT 2-COMPARISON TARGET PATTERN DETECTION AND IDENTIFICATION}

After seeing the subjects' performance in identifying the correct formant pattern, one is compelled to ask the question just how detectible the patterns were. This question was put to test in Experiment 2 in which detectability of a small number of selected target patterns was measured when its audibility was masked by the distracter used in Experiment 1. Figure 3 illustrates with the closed symbols data of the detection experiment. The same subjects' results in the identification experiment at the same conditions are also shown for comparison with the open symbols. 

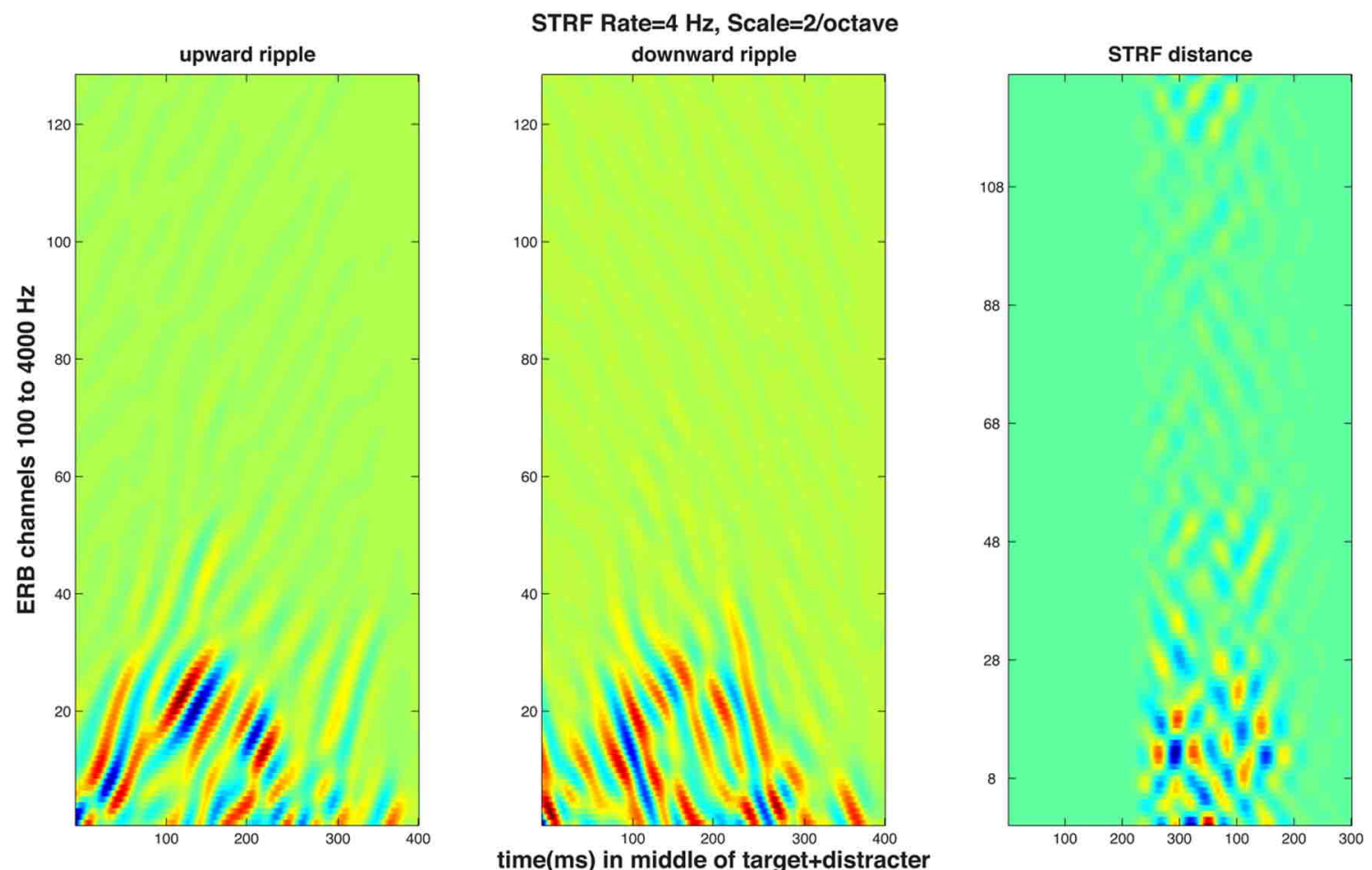

FIGURE 4 | STRF magnitude responses to a target-plus-distracter stimulus. The graphs represent simulated cortical response patterns of 128 channels tuned to frequencies aligned on an equivalent rectangular bandwidth (ERB) scale, as a function of time over the 600-ms middle portion of the stimulus containing 600-ms of the distracter and $400 \mathrm{~ms}$ of the target (see Figure 1) starting $100 \mathrm{~ms}$ after the portion of the distracter shown. All panels refer to STRF activity of regions tuned to $4-\mathrm{Hz}$ temporal modulation rate and 2 cycles/octave spectral modulation. Left and middle panels: upward (left) and downward (middle) time-frequency modulation ripple STRF responses to an up-down target pattern embedded in the distracter, i.e., activity elicited by $+4 \mathrm{~Hz}$ and $-4 \mathrm{~Hz}$ temporal modulation, respectively. The rightmost panel displays pixel-by-pixel Euclidean distances of STRF magnitude evoked by the up-down and the down-up target in an identical distracter, i.e., two stimuli differing only in the very center of their duration.
Elderly subjects needed the target level to be $18.6 \mathrm{~dB}$ higher than the young at the $60 \mathrm{~dB}$ SPL distracter level and $24.22 \mathrm{~dB}$ higher at the $80 \mathrm{~dB}$ SPL distracter level. At the easy (55\% swing) conditions detection of the target for the young subjects required a substantially lower (between 8.1 and $11.6 \mathrm{~dB}$ ) target level than did its identification, whereas for the elderly subjects the two tasks required the same level, except at the most intense distracter level, where identification level was $3.5 \mathrm{~dB}$ higher than the detection level. Contrary to identifiability of the target (as seen in Experiment 1), the extent of formant swing did not change its detectability: the swing had no influence on whether the target could be heard. Analysis of variance of these results, shown in Table 2, uncovered highly significant main effects and, except for distracter level, also within subject-main effect interactions. The highly significant subject group- task (detection vs. identification) and individual subject-task interactions indicate that a definite age effect for the way detection and identification are performed (and perhaps also understood). The significant groupformant swing and group-distracter level interactions show that elderly and young listeners are differentially affected by the difficulty of the task, be it detection or identification. The highly significant task-by-swing and task-by-distracter level interactions mean that factors making identification easier or more difficult had no bearing on detection, i.e., once a target was audible, many of its properties were irrelevant. This conclusion seems to have been shared by the two groups, as indicated by the non-significant subject group-by-task interaction.

\section{DISCUSSION}

Formant transitions in vowels convey important information. Although a large excursion of transition indicates phonemic change and mostly signals the presence of a diphthong, even a relatively minor dynamic change in the frequency of a formant peak contributes to intelligibility because it is one of the markers of the consonant preceding and following the vowel (Hillenbrand et al., 2001; Fogerty and Kewley-Port, 2009). The present experiments investigated the identifiability and detectability of a simplified form of these transitions in the presence of intense distracter transitions, both in the range of those indicating phonemic changelike the 55\% excursions-and those signaling the identity of preceding/following consonants-like the 10-20\% excursions. The FM rate chosen, $5 \mathrm{~Hz}$, was also similar to syllabic rate and thus makes the results comparable to speech and to the CPE. From a psychoacoustic standpoint, the results complement the vast and detailed body of information on modulation interference and modulation masking that has established parametric limits for detection and discrimination thresholds of a target in the presence of similar interference, with respect to differences 


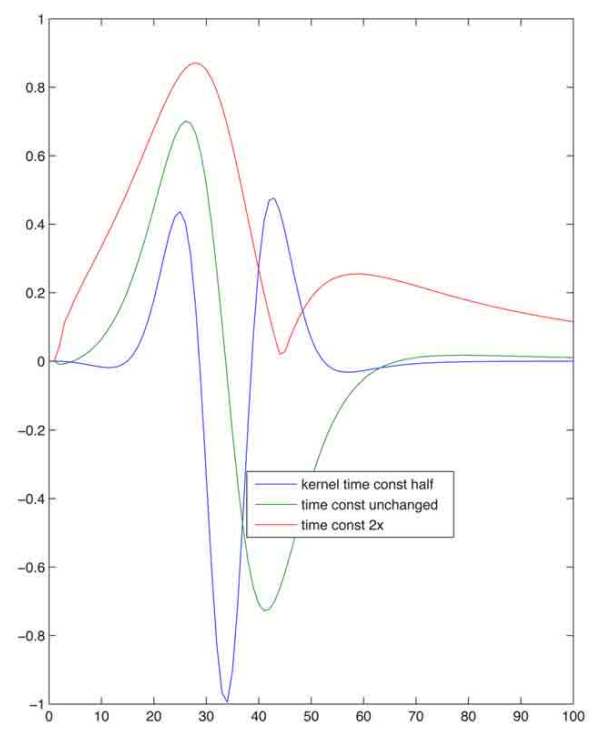

FIGURE 5 | Kernel functions used by the model of Chi et al. (2005) to generate temporal (left) and spectral (right) modulation planes at any given temporal rate and spectral scale specified. The line in the center of each of the three shown in the two panels is the unmodified kernel function and was used to simulate results of the young subjects. In both panels the lines

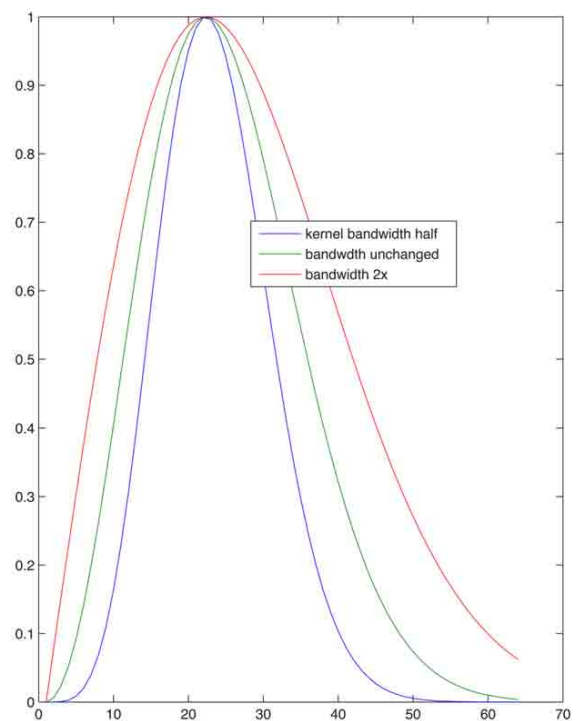

showing functions narrower than the central one were modified to produce temporal or spectral resolutions higher than those that supposedly underlay the good performance by the young listeners, whereas the lines outlining a broader function were modified to produce lower temporal or spectral resolutions that were expected to simulate results of the elderly subjects. in frequency, modulation rate, level, and some other properties. Although adding to this body was not the primary objective of the present study, it showed that increasing the level of interference resulted in a compressed growth in the level of the target, not only in the identification but also in the detection task. The present experiments used vowel-like harmonic target and distracter-a situation treated by only a relatively small number of studies (e.g., Shackleton and Carlyon, 1994; Lyzenga and Carlyon, 1999, 2005) that examined frequency modulation masking (FMD) for signals with f0's typically closer to each other than even those of our narrow f0 separation. Present in Lyzenga's and Carlyon's data, although not specifically pointed out in their papers, was the finding that FMD was larger when the target f0 was below that of the interferer. The present data shown in Figure 2 clearly show a worse overall performance, especially in the difficult $10 \%$ swing conditions, when the target f0 was lower than the distracter's. One explanation could be that when the distracter $\mathrm{f} 0$ is higher, it will have more intense harmonics in the frequency range where the target's second-to fifth harmonics (those that are most important for carrying pitch information) are located. Western composers from the Renaissance period on (i.e., from the beginnings of accompanied melody and polyphony) have been well aware of this relationship and have customarily placed the melody intended to be heard in the treble.

Clearly, the important finding of the experiments is the impaired ability by the elderly listeners to detect and identify formant excursions in the target embedded in a distracter. Since deficits have been documented for a variety temporal processing tasks in elderly individuals with little or no presbycusic impairment (Gordon-Salant and Fitzgibbons, 1999; Humes et al., 2012), it is unlikely that our elderly listeners' deficiency may be due to the presence of their not more severe than mild-to-moderate hearing loss. The surprising finding is the large difference, $20 \mathrm{~dB}$ on the whole, between target identification thresholds of young and elderly subjects. Thus, one could hypothetically assume that, in addition to a small part attributable to high-frequency threshold elevation that would have diminished the contribution of higher harmonics to the strength of the definition of formants, decline of a more central, possibly cortical, site may account for the observed perceptual loss.

\section{RELEVANCE OF THE RESULTS}

Formant transitions in vowels convey important information. Although a large excursion of transition indicates phonemic change and mostly signals the presence of a diphthong, even a relatively minor dynamic change in the frequency of a formant peak contributes to intelligibility because it is one of the markers of the consonant preceding and following the vowel (Hillenbrand et al., 2001; Fogerty and Kewley-Port, 2009). The present experiments investigated the identifiability and detectability of a simplified form of these transitions in the presence of intense distracter transitions, both in the range of those indicating phonemic change-like the 55\% excursions-and those signaling the identity of preceding/following consonants-like the 10-20\% excursions. The FM rate chosen, $5 \mathrm{~Hz}$, was also similar to syllabic rate and thus makes the results comparable to speech and to the CPE. From a psychoacoustic standpoint, the results complement the vast and detailed body of information on modulation interference and modulation masking that has established parametric limits for detection and discrimination thresholds of a target in the presence of similar interference, with respect to differences in frequency, modulation rate, level, and some other 


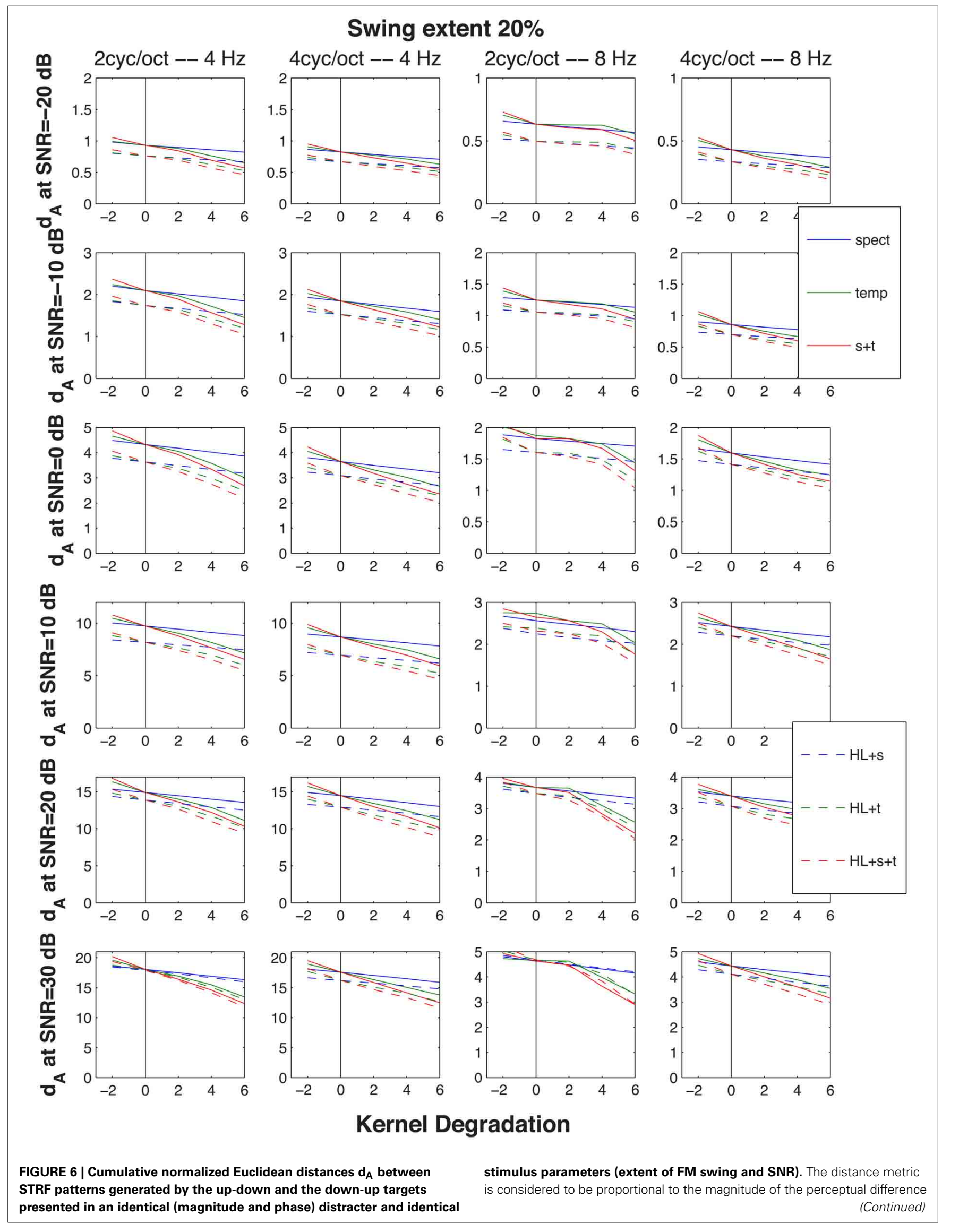




\section{FIGURE 6 | Continued}

between the two targets, hence its increasing magnitude with SNR indicated in the six rows of graphs. The abscissa indicates the size of degradation (i.e., the size of change of the kernel function): $6 \mathrm{~dB}$ corresponds to a 2-fold increase in the function's broadness and the vertical line at 0 marks the point at which the original, un-modified kernel functions were used, resulting in identification performance as good as demonstrated by the young subjects. The lines with negative slope show simulation by modification of the functions affecting resolution of the spectral, the temporal, or both the spectral and temporal modulations. Solid lines were generated with only the STRF kernels modified, whereas the broken lines resulted from entering the STRF module with a stimulus low-pass filtered and with a slightly reduced frequency selectivity, both intended to reflect changes encountered in elderly individuals with a moderate presbycusic sensorineural hearing loss. The leftmost tail of the lines indicate a point generated by an improved, rather than degraded, kernel function-hence the higher distance (e.g., better identification performance) it is associated with. The four columns represent the four combinations of two temporal modulation rates $(4$ and $8 \mathrm{~Hz}$ ) and two spectral modulation scales (2 and 4 cycles/octave). Across all conditions in the figure a single extent of FM excursion, $20 \%$ maximum counting from the $1 \mathrm{kHz}$ resting formant frequency, was used. properties. Although adding to this body was not the primary objective of the present study, it showed that increasing the level of interference resulted in a compressed growth in the level of the target, not only in the identification but also in the detection task. The present experiments used vowel-like harmonic target and distracter-a situation treated by only a relatively small number of studies (e.g., Shackleton and Carlyon, 1994; Lyzenga and Carlyon, 1999, 2005) that examined FMD for signals with f0's typically closer to each other than even those of our narrow f0 separation. Present in Lyzenga's and Carlyon's data, although not specifically pointed out in their papers, was the finding that FMD was larger when the target fo was below that of the interferer. The present data shown in Figure 2 clearly show a worse overall performance, especially in the difficult $10 \%$ swing conditions, when the target $\mathrm{f} 0$ was lower than the distracter's. One explanation could be that when the distracter fo is higher, it will have more intense harmonics in the frequency range where the target's second-to fifth harmonics (those that are most important for carrying pitch information) are located. Western composers from the Renaissance period on (i.e., from the beginnings of accompanied melody and polyphony) have been well aware of this relationship and have customarily placed the melody intended to be heard in the treble.

A comparison of the detection and identification results seen in Figure 3 would be interpreted by some authors (e.g., Brungart et al., 2001) as a contrast between energetic masking and informational masking-energetic masking being considered as the process underlying detection and informational masking as a process of interference not attributable to energetic masking (Durlach et al., 2003a). One particular result, however, is incompatible with the energetic-informational masking contrast: while young listeners needed a higher SNR for identification than for detection regardless of the difficulty of the task, for the easiest condition (when the target has the highest extent of FM swing) the older listeners needed the same SNR for detection and identification.

Clearly, the major finding of the experiments is that the ability by the elderly listeners to identify and also to detect formant excursions in the target embedded in a distracter is impaired. This finding adds to a long list of deficits for a variety temporal processing tasks in elderly individuals who, just as our elderly listeners, had little or no presbycusic impairment (e.g., Gordon-Salant and Fitzgibbons, 1999; Humes et al., 2012). While peripheral auditory impairment could have had some contribution to the $20 \mathrm{~dB}$ effect in our results even if the threshold shifts indicated by the elderly person's audiogram were relatively minor, it is likely that some dysfunction higher up on the auditory pathway was more accountable for the loss illustrated in Figure 2. Obviously, it would be of interest to answer the question regarding what proportion of the loss observed in the data is attributable to peripheral and what to central impairment. This question could be addressed empirically by conducting a series of tests on the same subjects to measure a wide range of spectral and temporal auditory capabilities that, according to the literature, could differentiate peripheral and central auditory processingsuch as amplitude and frequency modulation transfer functions, auditory filter width, pitch discrimination and salience, temporal processing in the 100-ms and longer ranges, auditory attention, and short-term memory for auditory stimulus details, only to cite a few. Unfortunately, such multidimensional data are not available for the subjects tested in the present experiments and the question can't be answered by analyzing the present data. Borrowing from physics, questions such as ours may be addressed indirectly by computational experiments using simulation. In our case, we could simulate normal and impaired processing of the present stimuli by using a model of the auditory system that includes both peripheral and central stages. The following subsection describes such a simulation with the help of tmodel mentioned in the Introduction, the STRF model (Chi et al., 2005). This model was chosen because it includes a peripheral and a central auditory stage, and because it permits manipulation of the efficacy of both stages.

\section{SIMULATION OF THE RESULTS USING THE STRF MODEL}

The STRF model first performs a multichannel filtering and compression akin those that take place in the cochlea and the auditory nerve, resulting in an "auditory spectrogram" with a critical band-type ERB (equivalent rectangular band) frequency scale (Patterson and Moore, 1986). This time-frequency response matrix is led to a subsequent stage in which temporal and spectral modulations are analyzed and decomposed to obtain a fourdimensional representation (time, frequency, temporal modulation by the rate of change, and spectral modulation by the scale of adjacent peaks in the spectrum). Such decomposition is known to take place in the cortex of animals (Kowalski et al., 1996) and humans (Mesgarani et al., 2008). The model is well suited for simulation of normal and impaired auditory processing on both levels because changing the threshold and the filtering in the first stage can mimic, to some extent, high-frequency presbycusic loss by the elderly. In the second stage, resolution of temporal and/or spectral modulation (i.e., grating) can be reduced by changing 


\section{SNR $0 \mathrm{~dB}$}

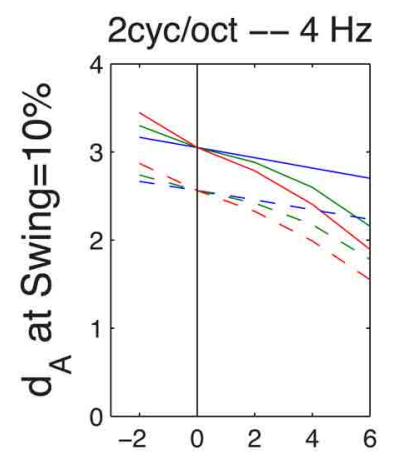

4 cyc/oct $--4 \mathrm{~Hz}$
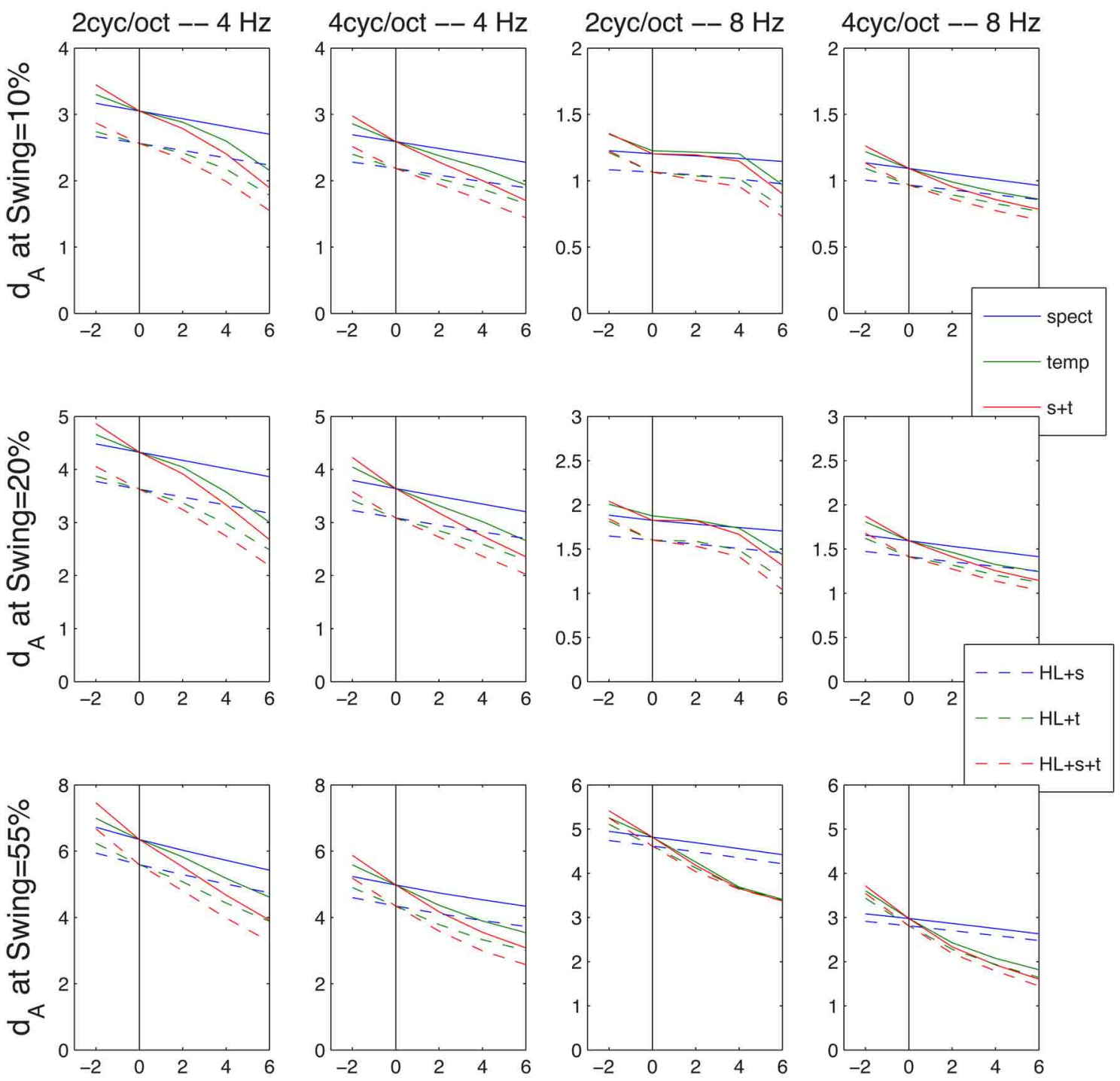

Kernel Degradation

FIGURE 7 | Similar to Figure 6, except that the cumulative normalized Euclidean distances $d_{A}$ between STRF patterns generated by the up-down and the down-up targets were evoked by the same target-distracter SNR of $0 \mathrm{~dB}$, across three $\mathrm{FM}$ excursion extents $(10,20$, and $55 \%$ maximum deflection re/the 1-kHz resting formant frequency), one for each row of graphs. The abscissa and the ordinate are the same as in Figure 6 (distance vs. size of kernel degradation). The four columns of graphs represent the same four rate-cycle combinations $(4,8 \mathrm{~Hz}$ and 2, 4 cycles) as in Figure 6. the appropriate parameters. Because of the magnitude of the effect obtained in the results, simulation of only the identification data was performed. It was assumed that identification of the up-down or the down-up pattern was based on the subject computing a distance between the four-dimensional STRF activity evoked by the two targets presented in the distracter. Since only one of the patterns was actually heard, it was further assumed that the STRF of the other target pattern was preserved and kept intact in memory, and it was available for the subject to perform the distance computation between the just-heard "pattern 1 " and the previously hear "pattern 2." To perform a simulated psychophysical experiment, STRF distances could have been computed on a series of repeated trials using a distracter presented starting with a random modulating phase and a d' statistic could have been calculated from the distribution of the trial-by-trial distances. Such simulation, unfortunately, would have required computational resources that were not available. As a substitute, distances were computed between STRF patterns generated by the two targets embedded into a distracter having fixed magnitude and phase spectra. The targets were the single-formant FM patterns used in the experiments and illustrated in Figure 1; three of the five FM excursions used in the experiments were used in the 


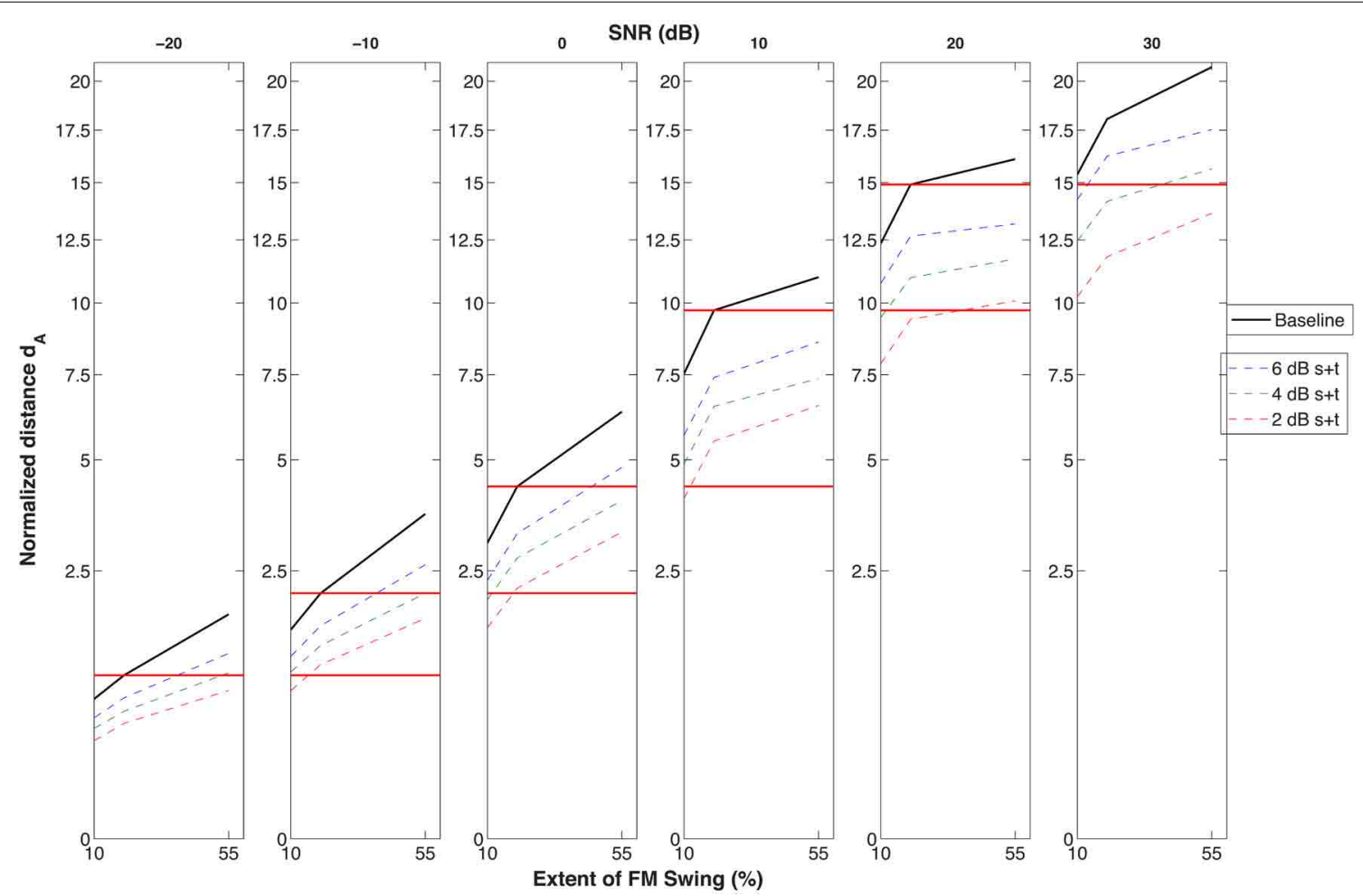

FIGURE 8 | Data simulating the almost-full set of conditions used in the identification experiments: identifiability of the targets (i.e., the cumulative normalized Euclidean distance between the STRF's evoked by the targets in distracter) as a function of three FM swing extents $(\mathbf{1 0}, \mathbf{2 0}$, and $\mathbf{5 5 \%})$ across six SNR's. The figure wishes to illustrate that, according to this simulation at least, the distance obtained by the un-degraded STRF (simulating the young subject) at he $20 \%$ swing extent in the -20 to $+20 \mathrm{~dB}$ SNR conditions is approximately equivalent to the distance obtained at a SNR $10 \mathrm{~dB}$ higher by the simulated elderly having a moderate presbycusic hearing loss and STRF's generated by spectral and temporal kernels twice their normal size, i.e., kernels that produced a markedly reduced resolution of both spectral and temporal modulations. This $10 \mathrm{~dB}$ loss, marked by the horizontal red lines, goes in the direction indicated by the experimental results shown in Figure 2, although it does not reach the $20 \mathrm{~dB}$ difference obtained in the psychophysical experiments. simulation $(10,20$, and 55\% formant peak change with respect to the $1-\mathrm{kHz}$ resting formant peak); the f0 of the target was always higher than that of the distracter and a single fundamental frequency difference $\Delta \mathrm{f} 0$ of 0.77 (the larger of the two tested in the experiments) was used. Figure 4 illustrates STRF time-frequency response patterns to the $55 \%$ targets in the distracter presented at $0 \mathrm{~dB}$ SNR. The two STRF's at the left show the upward and downward grating responses to the up-down pattern, whereas the rightmost panel shows time-frequency distances between the up-down and the down-up targets. Because the two stimuli differed only in their middle $200 \mathrm{~ms}$ portions (see Figure 1), the time range in the pictures contains $400 \mathrm{~ms}$ starting $100 \mathrm{~ms}$ before and finishing $100 \mathrm{~ms}$ after the FM portion of the targets.

Since the objective of this simulation was to compare the model's predictions for normal and impaired listeners, auditory processing by elderly individuals was modeled in two ways. First, the typical high-frequency sloping hearing loss was emulated by passing the stimulus through a low-pass filter with a $1600-\mathrm{Hz}$ corner frequency and a $6 \mathrm{~dB}$ /octave slope. In addition, the auditory spectrogram's filtering was made 10 percent less sharp, in order to mimic the broadening of cochlear frequency response often associated with age (Sommers and Gehr, 1998). Second, the spread of temporal (Takahashi and Bacon, 1992) and spectral (Sabin et al., 2013) modulation filters in aging was emulated by broadening the modulation filter kernels [the analogs to the Gabor (1946) transform's kernel] in the STRF model. This broadening of the two kernels is illustrated in Figure 5. For the data simulation, three different degrees of broadening (corresponding to factors of $1.26,1.56$, and 2, i.e., 2,4 , and $6 \mathrm{~dB}$ ) and one degree of sharpening (a factor of 0.8 , i.e., $-2 \mathrm{~dB}$ ) was used, either for the spectral, for the temporal, or both the spectral and temporal modulation filters. Results of these operations are illustrated in Figure 6 showing $\mathrm{d}_{\mathrm{A}}$, the normalized (using standard deviations) cumulative Euclidean distance measure. These distances were computed between corresponding pixels of the time-frequency plane across SNR's ranging from -20 to $30 \mathrm{~dB}$ at one selected FM swing (20\%) and are shown as a function of the degree of modulation filter degradation (with one negative degradation, i.e., improvement, as the leftmost point on each graph). The four columns of the figure display the distances for the four combinations of two the degrees of temporal modulation $(2$ and $4 \mathrm{~Hz})$ and two degrees pf spectral modulation ( 2 and 4 cycles/octave). These modulation degrees were seen as being the most sensitive to the stimuli used in the study. Each graph illustrates the effect of two sets of 
simulations, one (solid lines) for an intact first stage (=auditory spectrogram) input to the STRF stage, and one (broken lines) for the case in which the first stage contained a presbycusic analog low-pass filter. The difference between no-hearing-loss solid lines and the presbycusic-loss broken lines gauges the effect of the simulated peripheral hearing loss, best seen where they cross the vertical line indicating the condition in which the modulation filters were left undegraded. Such peripheral loss effect is present across all SNR's and all four rate/cycle combinations, although its size varies (between about 5\% to more than 25\%) and it is generally smaller for large SNR's. Comparing the three types of modulation filter degradation, combined widening of the spectral and temporal filters was the most destructive, widening of the spectral filter alone had the least effect, and widening of the temporal filter alone took place in the middle. The largest degradation effect, over $50 \%$, is associated with a $6 \mathrm{~dB}$ (i.e., doubled) increase of the parameter controlling spectral and temporal modulation filter width was seen for high-SNR low-pass filtered (i.e., presbycusic) stimuli. Similar observations can be made when looking at Figure 7 in which distance metrics across the three FM excursion extents are shown at the $0 \mathrm{~dB}$ SNR condition. Due to the relatively quiet signal level, absolute distance magnitudes and degradation effects are smaller than those seen in Figure 6.

Although the methods used and the $d_{A}$ metric adopted were not optimally suited for simulating psychometric functions, a graphic projection of the effect of degradation on SNR was attempted in Figure 8. In this figure the $d_{A}$ scale was used to allow comparison of data across SNR's and across the three FM excursion conditions (10,20, and 55\%). The performance of presbycusic-filtered inputs to the second stage that underwent the three types of degradation (broken lines) is compared with the no-filtering-no-degradation condition taken as the baseline (dark black line, emulating our young subjects). The $20 \%$ excursion taken as the criterion projected to the next, easier $10 \mathrm{~dB}$ higher SNR condition shows that although that particular performance level is exceeded by the baseline, it is within the range of degraded STRF processors. For instance, we see that a $-10 \mathrm{~dB}$ SNR for the most degraded (thin red line) condition with an (interpolated) excursion between 10 and $20 \%$ will lead to a performance level identical to that of a $10 \mathrm{~dB}$ less loud stimulus at a $20 \% \mathrm{FM}$ swing going through an intact (unfiltered/un-degraded) model. Similar $10 \mathrm{~dB}$ (or near $10 \mathrm{~dB}$ ) effects comparing the baseline with the most degraded condition can be seen at all SNR's. While this difference between undegraded-normal and degraded-impaired simulated subjects is smaller than the $20 \mathrm{~dB}$ drop in performance by the elderly compared to the young in the experiments, it still suggests that a degradation of the cortical processor responsible for modulation filtering may at least partially account for the age effect seen.

Aside the age effect the objective was to simulate, there are some valuable hints offered by the STRF model data. As expected, one can see in both Figures 6, 7 that those stimuli that easier to discriminate (such as larger SNR's and larger FM swings) produce larger or much larger distances. There is, however, a potentially more important observation. Although the stimuli were not speech, they were tailored with parameters reflecting the spectral and temporal dynamics of speech. Thus, it may not be without relevance to speech processing in the cortex that the largest distances were seen for the $4-\mathrm{Hz}$ temporal modulation filter at both the 2-and the 4-cycles/octave spectral modulation filter. This indicates that the most active temporal modulation filter coincides with the most prominent $4-\mathrm{Hz}$ modulation rate observed for conversational speech across talkers and across languages (Greenberg et al., 2003) and that the 2-to-4-cycles/octave spectral grating coincides with distances between peaks in the spectrum that are optimal for resolving formants and formant changes in vowels (Kewley-Port and Zheng, 1999).

Despite the fact that the simulation discussed in this subsection provides only an imperfect analogy to a psychophysical experiment, it has been able to provide an answer to the question that led us to emulating the data presented in the previous sections with the help of a well-established model - the STRF-that includes peripheral and central stages of auditory processing. This simulation appears to suggest that the deficit shown by the elderly listeners in the experiments is due to two factors. The first is a peripheral loss affecting frequency and temporal resolution, but only to a lesser degree than the second. This second, more potent factor is a deficiency in the resolution of temporal and spectral modulations performed by the auditory system at a more central site, most likely in the cortex. To better understand the role of this brain mechanism in the perception of everyday speech, work should be directed toward extracting principal features of STRF activity and the trajectory of those features over time. Such future work would allow us to get a better grip on mechanisms likely to be responsible for the CPE and its decay with age.

\section{ACKNOWLEDGMENTS}

The author wishes to thank Alex Brandmeyer, Kara Haupt, and J. C. Sander for the help they provided during the experimental phase of the study, René Carré and Steven Greenberg for many helpful discussions on speech dynamics, Lakshmi Krishnan and Nima Mesgarani for their help in the STRF analysis of the signals, and the three reviewers for their very helpful comments and suggestions. The research was supported by grant AG-07998 from the National Institute on Aging and by the Veterans Affairs Medical Research.

\section{SUPPLEMENTARY MATERIAL}

The Supplementary Material for this article can be found online at: http://www.frontiersin.org/journal/10.3389/fnins. 2014.00144/abstract

\section{Audio examples_to be listened to while looking at Figure 1.}

Audio example 1 | Ascending-descending target formant FM pattern (left pattern of top trace in Figure 1). High fundamental frequency $(189 \mathrm{~Hz})$ with an excursion wider than the widest used in the experiment $(75 \%)$. The $\mathrm{FM}$ rate is $5 \mathrm{~Hz}$.

Audio example 2 | Similar, but for the descending-ascending target formant FM pattern (right pattern of top trace in Figure 1).

Audio example 3 | 5- Hz FM pattern of the distracter. Low fundamental frequency $(107 \mathrm{~Hz})$. The formant swing is between the 525 and $100 \mathrm{~Hz}$ peak frequencies.

Audio example 4 | A waveform similar to whose presented to the listener in the identification experiment (Experiment 1). It is, actually, the pattern 
of Audio example 2 presented in the middle of the distracter. The SPLs of the target and of the distracter in this example are identical. This large target-to-distracter ratio, together with the target's formant swing larger than those used in the experiment, produce a stimulus in which the target pattern would have been easily identified by all listeners in the study.

\section{REFERENCES}

Abel, S. M., Giguere, C., Consoli, A., and Papsin, B. C. (2000). The effect of aging on horizontal plane sound localization. J. Acoust. Soc. Am. 108, 743-752. doi: $10.1121 / 1.429607$

Agus, T. R., Akeroyd, M. A., Gatehouse, S., and Warden, D. (2009). Informational masking in young and elderly listeners for speech masked by simultaneous speech and noise. J. Acoust. Soc. Am. 126, 1926-1940. doi: 10.1121/1.3205403

Arai, T., and Greenberg, S. (1997). "The temporal properties of spoken japanese are similar to those of English," in Paper presented at the Eurospeech (Rhodes).

Arbogast, T. L., Mason, C. R., and Kidd, J. G. (2002). The effect of spatial separation on informational and energetic masking of speech. J. Acoust. Soc. Am. 112, 2086-2098. doi: 10.1121/1.1510141

Assmann, P. F., and Summerfield, A. Q. (1990). Modeling the perception of concurrent vowels: vowels with different fundamental frequencies. J. Acoust. Soc. Am. 88, 680-697. doi: 10.1121/1.399772

Bacon, S. P., and Takahashi, G. A. (1992). Overshoot in normal-hearing and hearing-impaired subjects. J. Acoust. Soc. Am. 91, 2865-2871. doi: $10.1121 / 1.402967$

Bregman, A. S. (1990). Auditory Scene Analysis: The Perceptual Organization of Sound. Cambridge, MA: Bradford Books (MIT Press).

Brungart, D. S., and Simpson, B. D. (2002). Within-ear and across-ear interference in a cocktail-party listening task. J. Acoust. Soc. Am. 112, 2985-2995. doi: $10.1121 / 1.1512703$

Brungart, D. S., and Simpson, B. D. (2007). Effect of target-masker similarity on across-ear interference in a dichotic cocktail-party listening task. J. Acoust. Soc. Am. 122, 1724. doi: 10.1121/1.2756797

Brungart, D. S., Simpson, B. D., Ericson, M. A., and Scott, K. R. (2001). Informational and energetic masking effects in the perception of multiple simultaneous talkers. J. Acoust. Soc. Am. 110, 2527-2538. doi: 10.1121/1. 1408946

Carhart, R., and Tillman, T. W. (1970). Interaction of competing speech signals with hearing losses. Arch. Otolaryngol. 91, 273-279. doi: 10.1001/archotol.1970. 00770040379010

Chi, T., Ru, P., and Shamma, S. A. (2005). Multiresolution spectrotemporal analysis of complex sounds. J. Acoust. Soc. Am. 118, 887-906. doi: 10.1121/1.1945807

Cooke, M., Garcia Lecumberri, M. L., and Barker, J. (2008). The foreign language cocktail party problem: energetic and informational masking effects in non-native speech perception. J. Acoust. Soc. Am. 123, 414-427. doi: 10.1121/1. 2804952

Darwin, C. J., Brungart, D. S., and Simpson, B. D. (2003). Effects of fundamental frequency and vocal-tract length changes on attention to one of two simultaneous talkers. J. Acoust. Soc. Am. 114, 2913-2922. doi: 10.1121/1.1616924

Darwin, C. J., and Hukin, R. W. (1998). Perceptual segregation of a harmonic from a vowel by interaural time difference in conjunction with mistuning and onset asynchrony. J. Acoust. Soc. Am. 103, 1080-1084. doi: 10.1121/1.421221

Divenyi, P. (2005). "Masking the feature-information in multi-stream speechanalogue displays," in Speech Separation by Humans and Machines, ed P. Divenyi (New York, NY: Kluwer Academic Publishers), 269-281.

Divenyi, P. (2009). Perception of complete and incomplete formant transitions in vowels. J. Acoust. Soc. Am. 126, 1427-1439. doi: 10.1121/1.3167482

Divenyi, P. L., and Haupt, K. M. (1997). Audiological correlates of speech understanding deficits in elderly listeners with mild-to-moderate hearing loss. I. Age and laterality effects. Ear Hear. 18, 42-61. doi: 10.1097/00003446-19970200000005

Dubno, J. R., Dirks, D. D., and Morgan, D. E. (1984). Effects of age and mild hearing loss on speech recognition in noise. J. Acoust. Soc. Am. 76, 87-96. doi: $10.1121 / 1.391011$

Durlach, N. I., Mason, C. R., Kidd, G. Jr., Arbogast, T. L., Colburn, H. S., and ShinnCunningham, B. G. (2003a). Note on informational masking. J. Acoust. Soc. Am. 113, 2984-2987. doi: 10.1121/1.1570435

Durlach, N. I., Mason, C. R., Shinn-Cunningham, B. G., Arbogast, T. L., Colburn, H. S., and Kidd, G. Jr. (2003b). Informational masking: counteracting the effects of stimulus uncertainty by decreasing target-masker similarity. J. Acoust. Soc. Am. 114, 368-379. doi: 10.1121/1.1577562

Elhilali, M., and Shamma, S. A. (2008). A cocktail party with a cortical twist: how cortical mechanisms contribute to sound segregation. J. Acoust. Soc. Am. 124, 3751-3771. doi: 10.1121/1.3001672

Elliott, L. L., Hammer, M. A., Scholl, M. E., and Wasowicz, J. M. (1989). Age differences in discrimination of simulated single-formant frequency transitions. Percept. Psychophys. 46, 181-186. doi: 10.3758/BF03204981

Elliott, T. M., and Theunissen, F. E. (2009). The modulation transfer function for speech intelligibility. PLoS Comput. Biol. 5:e1000302. doi: 10.1371/journal.pcbi. 1000302

Ezzatian, P., Li, L., Pichora-Fuller, K., and Schneider, B. (2011). The effect of priming on release from informational masking is equivalent for younger and older adults. Ear Hear. 32, 84-96. doi: 10.1097/AUD.0b013e3181ee6b8a

Fitzgibbons, P. J., and Gordon-Salant, S. (1994). Age effects on measures of auditory duration discrimination. J. Speech Hear. Res. 37, 662-670.

Fitzgibbons, P. J., and Gordon-Salant, S. (1998). Auditory temporal order perception in younger and older adults. J. Speech Lang. Hear. Res. 41, 1052-1060.

Fogerty, D., and Kewley-Port, D. (2009). Perceptual contributions of the consonantvowel boundary to sentence intelligibilitya). J. Acoust. Soc. Am. 126, 847-857. doi: $10.1121 / 1.3159302$

Freyman, R. L., Balakrishnan, U., and Helfer, K. S. (2004). Effect of number of masking talkers and auditory priming on informational masking in speech recognition. J. Acoust. Soc. Am. 115, 2246-2256. doi: 10.1121/1.1689343

Freyman, R. L., Balakrishnan, U., and Helfer, K. S. (2008). Spatial release from masking with noise-vocoded speech. J. Acoust. Soc. Am. 124, 1627-1637. doi: $10.1121 / 1.2951964$

Gabor, D. (1946). Theory of communication. J. Inst. Elec. Eng. 93, 429-457.

Gelfand, S. A., Ross, L., and Miller, S. (1988). Sentence reception in noise from one versus two sources: effect of aging and hearing loss. J. Acoust. Soc. Am. 83, 248-256. doi: 10.1121/1.396426

Gordon-Salant, S., and Fitzgibbons, P. J. (1999). Profile of auditory temporal processing in older listeners. J. Speech Lang. Hear. Res. 42, 300-311.

Gordon-Salant, S., Fitzgibbons, P. J., and Friedman, S. A. (2007). Recognition of time-compressed and natural speech with selective temporal enhancements by young and elderly listeners. J. Speech Lang. Hear. Res. 50, 1181-1193. doi: 10.1044/1092-4388 (2007/082)

Greenberg, S., Carvey, H. M., Hitchcock, L., and Chang, S. (2003). Temporal properties of spontaneous speech - A syllable-centric perspective. J. Phonetics 31, 465-485. doi: 10.1016/j.wocn.2003. 09.005

Greenwood, D. D. (1962). Approximate calculation of the dimensions of travelingwave envelopes in four species. J. Acoust. Soc. Am. 34, 1364-1369. doi: $10.1121 / 1.1918349$

Hawley, M. L., Litovsky, R. Y., and Culling, J. F. (2004). The benefit of binaural hearing in a cocktail party: effect of location and type of interferer. J. Acoust. Soc. Am. 115, 833-843. doi: 10.1121/1.1639908

He, N. J., Mills, J. H., Ahlstrom, J. B., and Dubno, J. R. (2008). Age-related differences in the temporal modulation transfer function with pure-tone carriers. J. Acoust. Soc. Am. 124, 3841-3849. doi: 10.1121/1.2998779

Herman, G. E., Warren, L. R., and Wagener, J. W. (1977). Auditory lateralization: age differences in sensitivity to dichotic time and amplitude cues. J. Gerontol. 32, 187-191. doi: 10.1093/geronj/32.2.187

Hillenbrand, J. M., Clark, M. J., and Nearey, T. M. (2001). Effects of consonant environment on vowel formant patterns. J. Acoust. Soc. Am. 109, 748-763. doi: $10.1121 / 1.1337959$

Humes, L. E., Dubno, J. R., Gordon-Salant, S., Lister, J. J., Cacace, A. T., Cruickshanks, K. J., et al. (2012). Central presbycusis: a review and evaluation of the evidence. J. Am. Acad. Audiol. 23, 635-666. doi: 10.3766/jaaa.23.8.5

Humes, L. E., Kidd, G. R., and Lentz, J. J. (2013). Auditory and cognitive factors underlying individual differences in aided speech-understanding among older adults. Front. Syst. Neurosci. 7:55. doi: 10.3389/fnsys.2013.00055

Humes, L. E., Watson, B. U., Christensen, L. A., Cokely, C. G., Halling, D. C., and Lee, L. (1994). Factors associated with individual differences in clinical measures of speech recognition among the elderly. J. Speech Hear. Res. 37, 465-474.

Ihlefeld, A., and Shinn-Cunningham, B. (2008). Spatial release from energetic and informational masking in a divided speech identification task. J. Acoust. Soc. Am. 123, 4380-4392. doi: $10.1121 / 1.2904825$ 
Kewley-Port, D., and Zheng, Y. (1999). Vowel formant discrimination: towards more ordinary listening conditions. J. Acoust. Soc. Am. 106, 2945-2958. doi: $10.1121 / 1.428134$

Kidd, G. Jr., Mason, C. R., Deliwala, P. S., Woods, W. S., and Colburn, H. S. (1994). Reducing informational masking by sound segregation. J. Acoust. Soc. Am. 95, 3475-3480. doi: 10.1121/1.410023

Kowalski, N., Depireux, D. A., and Shamma, S. A. (1996). Analysis of dynamic spectra in ferret primary auditory cortex. I. Characteristics of single-unit responses to moving ripple spectra. J. Neurophysiol. 76, 3503-3523.

Lavandier, M., and Culling, J. F. (2008). Speech segregation in rooms: monaural, binaural, and interacting effects of reverberation on target and interferer. J. Acoust. Soc. Am. 123, 2237-2248. doi: 10.1121/1.2871943

Lee, J. H., and Humes, L. E. (2012). Effect of fundamental-frequency and sentenceonset differences on speech-identification performance of young and older adults in a competing-talker background. J. Acoust. Soc. Am. 132, 1700-1717. doi: $10.1121 / 1.4740482$

Lee, T. Y., and Richards, V. M. (2011). Evaluation of similarity effects in informational masking. J. Acoust. Soc. Am. 129, EL280-EL285. doi: 10.1121/1.3590168

Levi, A., and Stark, H. (1983). Signal restoration from phase by projection onto a convex set. J. Opt. Soc. Am. 73, 810-822. doi: 10.1364/JOSA.73.000810

Lutfi, R. A. (1990). How much masking is informational masking? J. Acoust. Soc. Am. 88, 2607-2610. doi: 10.1121/1.399980

Lyzenga, J., and Carlyon, R. P. (1999). Center frequency modulation detection for harmonic complexes resembling vowel formants and its interference by offfrequency maskers. J. Acoust. Soc. Am. 105, 2792-2806. doi: 10.1121/1.426896

Lyzenga, J., and Carlyon, R. P. (2005). Detection, direction discrimination, and offfrequency interference of center-frequency modulations and glides for vowel formants. J. Acoust. Soc. Am. 117, 3042-3053. doi: 10.1121/1.1882943

McAdams, S. (1990). Interactions among cues contributing to concurrent sound segregation. J. Acoust. Soc. Am. 88, S146. doi: 10.1016/j.heares.2009.09.012

Mesgarani, N., David, S. V., Fritz, J. B., and Shamma, S. A. (2008). Phoneme representation and classification in primary auditory cortex. J. Acoust. Soc. Am. 123, 899-909. doi: 10.1121/1.2816572

Mesgarani, N., Thomas, S., and Hermansky, H. (2011). Toward optimizing stream fusion in multistream recognition of speech. J. Acoust. Soc. Am. 130, EL14-EL18. doi: $10.1121 / 1.3595744$

Micheyl, C., and Oxenham, A. J. (2010). Pitch, harmonicity and concurrent sound segregation: psychoacoustical and neurophysiological findings. Hear. Res. 266, 36-51. doi: 10.1016/j.heares.2009.09.012

Moore, B. C., Sek, A., and Shailer, M. J. (1995). Modulation discrimination interference for narrow-band noise modulators. J. Acoust. Soc. Am. 97, 2493-2497. doi: $10.1121 / 1.411969$

Moore, B. C. J., and Sek, A. (1996). Detection of frequency modulation at low modulation rates: evidence for a mechanism based on phase locking. J. Acoust. Soc. Am. 100, 2320-2331. doi: 10.1121/1.417941

Murphy, D. R., Craik, F. I., Li, K. Z., and Schneider, B. A. (2000). Comparing the effects of aging and background noise on short-term memory performance. Psychol. Aging 15, 323-334. doi: 10.1037/0882-7974.15.2.323

Ng, E. H., Rudner, M., Lunner, T., Pedersen, M. S., and Ronnberg, J. (2013). Effects of noise and working memory capacity on memory processing of speech for hearing-aid users. Int. J. Audiol. 52, 433-441. doi: 10.3109/14992027.2013.776181

Oh, E. L., and Lutfi, R. A. (1998). Nonmonotonicity of informational masking. J. Acoust. Soc. Am. 104, 3489-3499. doi: 10.1121/1.423932

Patterson, R., and Moore, B. (1986). "Auditory filters and excitation patterns as representations of frequency resolution," in Frequency Selectivity in Hearing, ed B. Moore (London: Academic Press), 123-178.

Pitton, J. W., Wang, K., and Juang, B.-H. (1996). Time-frequency analysis and auditory modeling for automatic recognition of speech. Proc. IEEE 84, 1199-1215. doi: $10.1109 / 5.535241$
Plomp, R. (1983). "Perception of speech as a modulated signal," in Proceedings of the 10th International Congress of Phonetic Science, M. P. R. van der Broecke and A. Cohen (Dordrecht: Foris), 29-40.

Rajan, R., and Cainer, K. E. (2008). Ageing without hearing loss or cognitive impairment causes a decrease in speech intelligibility only in informational maskers. Neuroscience. 154, 784-795. doi: 10.1016/j.neuroscience.2008. 03.067

Roman, N., and Wang, D. (2006). Pitch-based monaural segregation of reverberant speech. J. Acoust. Soc. Am. 120, 458-469. doi: 10.1121/1.2204590

Sabin, A. T., Clark, C. A., Eddins, D. A., and Wright, B. A. (2013). Different patterns of perceptual learning on spectral modulation detection between older hearing-impaired and younger normal-hearing adults. J. Assoc. Res. Otolaryngol. 14, 283-294. doi: 10.1007/s10162-012-0363-y

Shackleton, T. M., and Carlyon, R. P. (1994). The role of resolved and unresolved harmonics in pitch perception and frequency modulation discrimination. J. Acoust. Soc. Am. 95, 3529-3540. doi: 10.1121/1.409970

Singh, N. C., and Theunissen, F. E. (2003). Modulation spectra of natural sounds and ethological theories of auditory processing. J. Acoust. Soc. Am. 114, 3394-3411. doi: 10.1121/1.1624067

Snell, K. B., and Frisina, D. R. (2000). Relationships among age-related differences in gap detection and word recognition. J. Acoust. Soc. Am. 107, 1615-1626. doi: $10.1121 / 1.428446$

Snell, K. B., Mapes, F. M., Hickman, E. D., and Frisina, D. R. (2002). Word recognition in competing babble and the effects of age, temporal processing, and absolute sensitivity. J. Acoust. Soc. Am. 112, 720-727. doi: 10.1121/1.1487841

Sommers, M. S. (1997). Stimulus variability and spoken word recognition. II. The effects of age and hearing impairment. J. Acoust. Soc. Am. 101, 2278-2288. doi: $10.1121 / 1.418208$

Sommers, M. S., and Gehr, S. E. (1998). Auditory suppression and frequency selectivity in older and younger adults. J. Acoust. Soc. Am. 103, 1067-1074. doi: $10.1121 / 1.421220$

Takahashi, G. A., and Bacon, S. P. (1992). Modulation detection, modulation masking, and speech understanding in noise in the elderly. J. Speech Hear. Res. 35, 1413-1429.

Terhardt, E., Stoll, G., and Seewann, M. (1982). Algorithm for extraction of pitch and pitch salience from complex tonal signals. J. Acoust. Soc. Am. 71, 679-688. doi: $10.1121 / 1.387544$

Watson, C. S. (1987). "Uncertainty, informational masking, and the capacity of immediate auditory memory," in Auditory Processing of Complex Sounds, ed W. A. Yost and C. S. Watson (Hillsdale, NJ: L. Erlbaum), 267-287.

Zekveld, A. A., Kramer, S. E., and Festen, J. M. (2011). Cognitive load during speech perception in noise: the influence of age, hearing loss, and cognition on the pupil response. Ear Hear. 32, 498-510. doi: 10.1097/AUD.0b013e31820512bb

Conflict of Interest Statement: The author declares that the research was conducted in the absence of any commercial or financial relationships that could be construed as a potential conflict of interest.

Received: 13 February 2014; accepted: 22 May 2014; published online: 12 June 2014. Citation: Divenyi P (2014) Decreased ability in the segregation of dynamically changing vowel-analog streams: a factor in the age-related cocktail-party deficit? Front. Neurosci. 8:144. doi: 10.3389/fnins.2014.00144

This article was submitted to Auditory Cognitive Neuroscience, a section of the journal Frontiers in Neuroscience.

Copyright (C) 2014 Divenyi. This is an open-access article distributed under the terms of the Creative Commons Attribution License (CC BY). The use, distribution or reproduction in other forums is permitted, provided the original author(s) or licensor are credited and that the original publication in this journal is cited, in accordance with accepted academic practice. No use, distribution or reproduction is permitted which does not comply with these terms. 